\title{
THE CHARACTERISTIC RING AND THE "BEST" WAY TO ADJOIN A ONE
}

\author{
W. D. BURGESS and P. N. STEWART
}

(Received 19 February 1988)

Communicated by B. J. Gardner

\begin{abstract}
For any ring $S$ we define and describe its characteristic ring, $\kappa(S)$. It plays the rôle of the usual characteristic even in rings whose additive structure, $(S,+)$, is complicated. The ring $\kappa(S)$ is an invariant of $(S,+)$ and also reflects certain non-additive properties of $S$. If $R$ is a left faithful ring without identity element, we show how to use $\kappa(R)$ to embed $R$ in a ring $R^{1}$ with identity. This unital overring of $R$ inherits many ring properties of $R$; for instance, if $R$ is artinian, noetherian, semiprime Goldie, regular, biregular or a $V$-ring, so too is $R^{1}$. In the case of regularity (or generalizations thereof), $R^{!}$satisfies a universal property with respect to the adjunction of an identity
\end{abstract}

1980 Mathematics subject classification (Amer. Math. Soc.) (1985 Revision): 16 A 56, 16 A 30, 13 A 99.

The usual characteristic of a ring does not carry much information when the ring is not a domain. If $S$ is a ring with 1 , the subring maxepi $(\nu, S)$, where $\nu: \mathbf{Z} \rightarrow S$, is proposed as the characteristic ring, $\kappa(S)$, of $S$. It is an invariant of the additive structure of $S$ and its ring structure is known. It is here completely described in terms of $(S,+)$, but it reflects more than the additive structure since, for example, it is regular ( $\pi$-regular) if $S$ is. Some properties of $\kappa(S)$ are described and it is seen that it can reasonably be defined even for rings without 1 .

The authors' research was supported in part by grants A7539 and A8789, respectively, of the NSERC. The second author is grateful for the hospitality of the University of Ottawa where this paper was written while he was there on sabbatical leave. Both authors appreciate the referee's helpful comments.

(C) 1989 Australian Mathematical Society $0263-6115 / 89 \$ A 2.00+0.00$ 
It was observed as long ago as 1932 (Dorroh's Theorem) that any nonunital ring $R$ may be embedded in a ring with identity. This is done by adjoining a copy of $Z$, the ring of integers, to $R$. This does not preserve all the nice properties which $R$ might have, nor is it minimal in any of various senses; and so over the decades many embeddings have been invented to serve diverse purposes. For example, if $R$ is regular (or some generalization of regular, such as $\pi$-regular) one would like to embed $R$ into a regular ring (or the generalization). There are other sorts of properties (semiprime, artinian, domain, Ore domain) which one may wish to preserve in going from $R$ to some ring with 1 , say $R^{1}$, all the while without adjoining anything more than necessary. It turns out that there is one construction, using $\kappa(R)$, which will give all the main results as well as some new ones, although there is not yet one proof by which to do it. In the case of the generalized sorts of regularity, the ring formed by adding $\kappa(R)$ satisfies a universal property with respect to the adjunction of 1 .

\section{The characteristic ring}

The basis for what follows is what could well be called the characteristic ring of a ring $S$ with 1 (the expression was first used in [2] but the germ of the idea is in [8] and [9]). In order to describe it, it is first necessary to recall what is meant by an epimorphism of rings with $1: \phi: S \rightarrow T$ is called an epimorphism of rings if for any ring $U$ and any pair of homomorphisms $\alpha, \beta: T \rightarrow U, \alpha \phi=\beta \phi$ implies $\alpha=\beta$. Such homomorphisms are not necessarily surjective as $\mathbf{Z} \rightarrow \mathbf{Q} \times \mathbf{Z} /(2), z \rightarrow(z, \bar{z})$, which is epic, shows. If $f: S \rightarrow T$ there is always a maximal epic extension of $f(S)$ in $T$, denoted maxepi $(f, T)$. The claim is that for the canonical homomorphism $\nu: \mathrm{Z} \rightarrow S$, maxepi $(\nu, S):=\kappa(S)$ acts like the characteristic of $S$. Indeed, if $S$ is a division ring then $\kappa(S)$ is the prime field of $S$. On the other hand $\kappa(\mathbf{Q} \times Z /(2))=\mathbf{Q} \times \mathbf{Z} /(2)$, revealing the mixture of characteristics of that ring. The ring $\kappa(S)$ is always central [17, Proposition 1.3] and the structure of all epimorphs of $\mathbf{Z}$ is known (in fact of all epimorphs of an arbitrary Dedekind domain). (See [1] and [16] for the case of $Z$ and [3] and [5] for the generalization.)

Proposition 1.1 [3]. A ring with $1, S$, is an epimorph of $\mathrm{Z}$ if and only if it has one of the following forms.

(A) $S \cong \mathbf{Z} / I$, for some ideal I of $\mathbf{Z}$.

(B) $S \cong D \times \mathbf{Z} /\left(p_{1}^{n_{1}}\right) \times \cdots \times \mathbf{Z} /\left(p_{k}^{n_{k}}\right)$, where the $p_{i}$ are primes, the $n_{i} \geq 1$ and $D$ is a ring, with $\mathrm{Z} \subseteq D \subseteq \mathrm{Q}$, which is divisible by the $p_{1}, \ldots, p_{k}$. 
(C) There is an infinite set of primes $\left\{p_{i}\right\}_{i \in \mathbf{N}}$ and, for $i \in \mathbf{N}$, integers $n_{i} \geq 1$ and a ring $D, Z \subseteq D \subseteq \mathbf{Q}$, with $D$ divisible by each of the $p_{i}$, and $S$ is the subring of $\prod_{i=1}^{\infty} Z /\left(p_{i}^{n_{i}}\right)$ consisting of sequences of the form $\left(u_{i}\right)$, where there is an element $a / b \in D$ such that for almost all $i, u_{i}$ has the form $\bar{a} / \bar{b} \in \mathbf{Z} /\left(p_{i}^{n_{i}}\right)$. (One can think of sequences of the product which are eventually "constant" and "in" D.)

Note that in cases (B) and (C) the ring $S$ is determined by a function $g: \mathbf{P} \rightarrow \mathbf{N} \cup\{\infty\}$, where $g(p)=n$ if the factor $\mathbf{Z} /\left(p^{n}\right)$ appears and $g(p)=0$ if $D$ is $p$-divisible but no factor $Z /\left(p^{n}\right)$ appears for any $n>0$, and, finally, $g(p)=\infty$ otherwise. (See [5].)

As we know, even in the case of domains, the characteristic ring can change under homomorphisms; nevertheless a few observations are possible. The first is immediate from the definition of $\kappa(S)$ as a maximal epimorphic extension.

Lemma 1.2. Let $\phi: S \rightarrow T$ be a homomorphism of rings with 1 . Then $\phi(\kappa(S))$ is a subring of $\kappa(T)$.

In what follows, for any abelian group $G, t(G)$ denotes its torsion subgroup, while for any $p \in \mathbf{P}, t_{p}(G)$ is its $p$-torsion part. In the case of a ring, these are all ideals. For any ring $S, \operatorname{ann}_{\mathrm{Z}} S$ denotes $\{z \in Z: z S=0\}$.

Proposition 1.3. Let $K$ and $L$ be epimorphs of $\mathbf{Z}$ where $K$ is a unital subring of $L$. Then

(i) $\operatorname{ann}_{\mathbf{Z}} K=\operatorname{ann}_{\mathbf{Z}} L$,

(ii) if $\operatorname{ann}_{\mathrm{Z}} K=\operatorname{ann}_{\mathrm{Z}} L=0$ then $K$ and $L$ are defined by functions $g_{K}, g_{L}$ : $\mathbf{P} \rightarrow \mathbf{N} \cup\{\infty\}$. For each $p \in \mathbf{P}$, if $g_{K}(p)<\infty$ then $g_{K}(p)=g_{L}(p)$. If $g_{K}(p)=\infty, g_{L}(p)$ may take on any value. Conversely, if $L$ is given by $g_{L}$, take any subset $U_{0}$ of $\mathbf{X}_{0}(L)=\left\{p \mid g_{L}(p)<\infty\right\}$, and define $g_{K}$ by

$$
g_{K}(p)= \begin{cases}g_{L}(p) & \text { if } p \in U_{0}, \\ \infty & \text { otherwise. }\end{cases}
$$

Then $g_{K}$ defines an epimorph of $\mathbf{Z}$ which is a subring of $L$.

Proof. Assume $K \subseteq L$. The first part is clear since $\operatorname{ann}_{\mathbf{Z}} K=\operatorname{ann}_{\mathbf{Z}}(1)=$ $\operatorname{ann}_{\mathrm{Z}} L$. If $\operatorname{ann}_{\mathrm{Z}} K=0$ and $p \in \mathbf{P}$ with $0<g_{K}(p)<\infty$ then $t_{p}(K) \neq 0$, and so $t_{p}(L) \neq 0$. Hence $0<g_{L}(p)<\infty$. Moreover, there is an idempotent $e_{p} \in L, e_{p} L=t_{p}(L)$, and an idempotent $f_{p}$ of $K$ with $f_{p} K=t_{p}(K)$. Then $f_{p}$ is a non-zero idempotent in the ring $t_{p}(L)$ which has only one, $e_{p}$, which generates it as a ring. Thus $t_{p}(K)=t_{p}(L)$, and $g_{K}(p)=g_{L}(p)$. If $g_{K}(p)=0$, 
$K$ is $p$-divisible and so $1 \in K$ has infinite $p$-height and hence $L$ is $p$-divisible and $g_{L}(p)=0$.

If $g_{K}(p)=\infty, g_{L}(p)$ can have any value. (Consider, for example, $Z[1 / 2] \times$ $\mathbf{Z} /(2) \rightarrow \mathbf{Z}[1 / 2,1 / 3] \times \mathbf{Z} /(2) \times \mathbf{Z} /(3),(1, \overline{0}) \rightarrow(1, \overline{0}, \overline{1})$ and $(0, \overline{1}) \rightarrow(0, \overline{1}, \overline{0})$.

The converse is clear: for if $\left(a_{p_{1}}, \ldots, d\right) \in K$ (either a finite sequence or an eventually "constant" sequence), this element can be sent to $\left(b_{q_{1}}, \ldots, d\right) \in L$ where

$$
b_{q_{i}}=\left\{\begin{array}{l}
a_{q_{i}} \text { if } q_{i} \in U_{0} \cap\left\{p \mid 0<g_{L}(p)<\infty\right\}, \\
\bar{q}, \text { the image of } d \text { in the } q_{i} \text {-component, otherwise. }
\end{array}\right.
$$

Finally, we can see something of what happens to the characteristic ring under a ring homomorphism

Proposition 1.4. Let $\phi: S \rightarrow T$ be a homomorphism of rings with 1. Then $\operatorname{ann}_{\mathrm{Z}} T \supseteq \operatorname{ann}_{\mathrm{Z}} S$. Further, if $\operatorname{ann}_{\mathrm{Z}} T=0$ then $\kappa(S)$ and $\kappa(T)$ are given by functions $g_{S}$ and $g_{T}$, respectively. In this case, for $p \in \mathbf{P}, g_{T}(p) \leq g_{S}(p)$.

Proof. The first part is clear. Assume that $\operatorname{ann}_{\mathrm{Z}} T=0$. If $g_{S}(p)<\infty$ then there is an idempotent $e_{p}$ (which could be 0 ) giving $t_{p}(S)=e_{p} S$ and $1-e_{p}$ has infinite $p$-height. If $x \in t_{p}(T), x=\phi\left(1-e_{p}\right) x+\phi\left(e_{p}\right) x$. Since $\phi\left(1-e_{p}\right)$ has infinite $p$-height, $\phi\left(1-e_{p}\right) x=0$. Thus $\phi\left(t_{p}(S)\right)=t_{p}(T)$. It follows that $g_{T}(p) \leq g_{S}(p)$. If $g_{S}(p)=\infty, g_{T}(p)$ can take on any value.

Of course if $R$ does not have 1 then $\kappa(R)$ is not defined but we shall see that it is nevertheless possible to see what it "should" be. To this end we first analyze the relationship between the characteristic ring of a ring with $1, S$, and its additive structure, $(S,+)$.

Proposition 1.5. Let $S$ be a ring with 1. Let $\mathbf{P}$ be the set of prime integers. There are two cases.

(i) $\operatorname{ann}_{\mathrm{Z}} S=I \neq 0$; in this case $\kappa(S) \cong \mathrm{Z} / I$.

(ii) $\operatorname{ann}_{\mathbf{Z}} S=0$. A function $f: \mathbf{P} \rightarrow N \cup\{\infty\}$ is defined as follows: if for $p \in \mathbf{P}, \operatorname{ann}_{\mathrm{Z}} t_{p}(S)=\left(p^{k}\right), k \geq 0$ and $S=S^{(p)} \oplus t_{p}(S)$ for some subgroup $S^{(p)}$ which is p-divisible, then $f(p):=k$; otherwise $f(p):=\infty$. Put $\{p \in \mathbf{P} \mid 0<$ $f(p)<\infty\}:=\mathbf{X}_{1}$ and $\{p \in \mathbf{P} \mid 0 \leq f(p)<\infty\}:=\mathbf{X}_{0}$. Then

(a) if $\mathbf{X}_{1}=\left\{p_{1}, \ldots, p_{k}\right\}$ is finite then $\kappa(S) \cong D \times \mathbf{Z} /\left(p_{1}^{f\left(p_{1}\right)}\right) \times \cdots \times \mathbf{Z} /\left(p_{k}^{f\left(p_{k}\right)}\right)$, where $\mathbf{Z} \subseteq D \subseteq \mathbf{Q}$ and $D=\mathbf{Z}\left[1 / p \mid p \in \mathbf{X}_{0}\right]$.

(b) if $\mathbf{X}_{1}$ is infinite then $\kappa(S)$ is the set of all sequences $\left\{u_{i}\right\}$ from $\prod_{i=1}^{\infty} \mathbf{Z} /\left(p_{i}^{f\left(p_{i}\right)}\right)$ which are eventually of the form $u_{i}=\bar{a} / \bar{b}$, where $a / b \in D=$ $\mathbf{Z}\left[1 / p \mid p \in \mathbf{X}_{0}\right]$. 
Conversely, $\kappa(S)$ has one of the forms of (1.1) and these show the structure of $(S,+)$ as follows: if we have form (A) then $\operatorname{ann}_{\mathrm{Z}}(S)=I$ and if forms (B) or $(C), \operatorname{ann}_{\mathrm{Z}}(S)=0$ and we get the function $f$ by $f\left(p_{i}\right)=n_{i}$ for the primes appearing in the product and $f(p)=0$ if $p$ does not appear in the product but $D$ is $p$-divisible; otherwise $f(p)=\infty$.

Proof. (i) If $\operatorname{ann}_{\mathrm{Z}}(S)=I \neq 0$ then the image of $\mathrm{Z}$ in $S$ is $\mathrm{Z} / I$, which is an artinian ring, and such rings have no proper epimorphic extensions [16, 5.4]. Conversely, if $\kappa(S) \cong \mathrm{Z} / I$, for some $I$, then clearly $\operatorname{ann}_{\mathrm{Z}} S=I$.

(ii) The case where $\operatorname{ann}_{\mathrm{Z}} S=0$.

Suppose that $\kappa(S)$ is of the form (B) or (C) as in (1.1). Then the idempotents of $\kappa(S)$ split $S$ exactly as in the statement of this theorem. Indeed if we take the function $g$ for $\kappa(S)$ then if $g(p)=k<\infty$ it follows that $\operatorname{ann}_{\mathrm{Z}} t_{p}(S)=\left(p^{k}\right)$, since this factor of $\kappa(S)$ is given by a central idempotent $e$ such that $1-e$ has infinite $p$-height. In other words, if $\kappa(S)$ is defined by the function $g$ then $g=f$.

In the other directions, set $\mathbf{X}_{1}=\left\{p_{1}, \ldots, p_{k}, \ldots\right\}$, which may be finite or infinite. For each $k$ there is a splitting

$$
S=S_{k} \oplus t_{p_{1}}(S) \oplus \cdots \oplus t_{p_{k}}(S)
$$

for some $S_{k}$ which is $p_{1}, \ldots, p_{k}$-divisible; the splitting is as a ring. To see that this is so, suppose that $S_{k-1}$ has been chosen and let $s \in t_{p_{k}}(S)$. Then if we write $f(k)$ for $f\left(p_{k}\right)$ we have $s=p_{1}^{f(1)} \cdots p_{k-1}^{f(k-1)} r$ for some $r \in S$, where $r=r_{1}+\cdots+r_{k-1}+t$ with $r_{i} \in t_{p_{i}}(S)$ and $t \in S_{k-1}$. Then if $m=p_{1}^{f(1)} \cdots p_{k-1}^{f(k-1)}$ we have $s=m r=m r_{1}+\cdots+m r_{k-1}+m t \in S_{k-1}$. Hence $t_{p_{k}}(S) \subseteq S_{k-1}$. But $m S=S_{k-1}$ and $S^{\left(p_{k}\right)}=p_{k}^{f(k)} S$ so if we put $S_{k}=m p_{k}^{f(k)} S$, then $S_{k} \subseteq S_{k-1}$, $t_{p_{k}}(S) \cap S_{k}=0$ and $S_{k-1}=S_{k} \oplus t_{p_{k}}(S)$.

Now let $T$ be the epimorph of $\mathbf{Z}$, defined by the function $f$, which we wish to show isomorphic to $\kappa(S)$. A homomorphism $\phi: T \rightarrow S$ can be defined as follows: let $t=\left(\bar{a}_{1}, \bar{a}_{2}, \ldots\right)$, with last entry $b / c \in D$ if $\mathbf{X}_{1}$ finite or otherwise with the sequence eventually, say after the $k$ th term, of the form $\bar{b} / \bar{c}$. Define $\phi(t):=\bar{a}_{1} e_{1}+\cdots+\bar{a}_{k} e_{k}+b s$ where $e_{i}$ is the idempotent giving $t_{p_{i}}(S)$ in the splitting $(*)$ and $s$ is the element of $S_{k}$ which solves $c s=1-\left(e_{1}+\cdots+e_{k}\right)$. This is possible since the prime factors of $c$ are among $p_{1}, \ldots, p_{k}$ and $S_{k}$ is divisible by all these primes. It is clear that $\phi$ is a ring homomorphism and if $\phi(t)=0$, then $\bar{a}_{1} e_{1}=\cdots=\bar{a}_{k} e_{k}=0$ giving $\bar{a}_{1}=\overline{0}, \ldots, \bar{a}_{k}=\overline{0}$; from this $b c s=b\left(1-\left(e_{1}+\cdots+e_{k}\right)\right)=0$ and $b S_{k}=0$. But $S_{k}$ is not torsion, so $b=0$.

It remains to show that $\phi(T)=\operatorname{maxepi}(\nu, S)=\kappa(S)$. But as already observed, the function $g$ which gives rise to $\kappa(S)$ is the same as $f$, and this shows that $\kappa(S)=\phi(T)$. 


\section{The basic construction}

Any ring $R$, which need not have a 1 , has either $\operatorname{ann}_{\mathrm{Z}} R \neq 0$ or $\operatorname{ann}_{\mathrm{Z}} R=0$ and then it is possible to define a function $f: \mathbf{P} \rightarrow \mathbf{N} \cup\{\infty\}$ just as in the statement of (1.5). hence associated to each ring $R$ is an epimorph of $\mathbf{Z}$, which is either $\mathbf{Z} / I$, for some ideal $I \neq 0$, or is given by the function $f$. Call this ring $\kappa(R)$ as well.

LEMMA 2.1. The ring $R$ is a $\kappa(R)-\kappa(R)$ bimodule.

Proof. If $\operatorname{ann}_{\mathrm{Z}} R=I \neq 0$ then $\kappa(R)=\mathrm{Z} / I$ and the action is obvious. In the other case, the action is based on the structure of $(R,+)$. Let $z=$ $\left(z_{1}, \ldots, z_{k}, \ldots\right)$, where the sequence is finite ending in $b / c \in D$ or it is infinite and eventually (say after $k$ terms) of the form $\bar{b} / \bar{c}$ where $b / c \in D$. If $r \in R$ then we write $r=r_{1}+\cdots+r_{k}+s$, where $r_{i} \in t_{p_{i}}(R)$, for $i=1, \ldots, k$ and $s \in R_{k}$. Here $R_{k}$ comes from the splitting of $R$ as in (*) in the proof of (1.5). Then $r z=z r:=z_{1} r_{1}+\cdots+z_{k} r_{k}+b t$, where the natural $\mathbf{Z} /\left(p_{i}^{f\left(p_{i}\right)}\right)$-structure on $t_{p_{i}}(R)$, for $i=1, \ldots, k$, is used and $t \in R_{k}$ solves $c t=s$. The remaining details are easy to verify.

This allows us to adjoin $\kappa(R)$ to $R$ in a direct way: $R^{*}:=R \times \kappa(R)$, with arithmetic $(r, z)+\left(r^{\prime}, z^{\prime}\right):=\left(r+r^{\prime}, z+z^{\prime}\right)$ and $(r, z)\left(r^{\prime}, z^{\prime}\right):=\left(r r^{\prime}+\right.$ $\left.z^{\prime} r+z r^{\prime}, z z^{\prime}\right)$. This ring would serve many of our purposes but it might be bigger than necessary. For example if $p \in \mathbf{X}_{1}$, it may be that $t_{p}(R)$ is already generated by a central idempotent, in which case the one contributed by $\kappa(R)$ is superfluous. We shall follow the lead of Robson [14] and look inside $\operatorname{End}\left(R_{R}\right)$, at least in the case where $R$ is left faithful, that is, if $r \in R$ is such that $r R=0$, then $r=0$. When $R$ is left faithful, $R$ embeds in $\operatorname{End}\left(R_{R}\right)$. Our proposed method of adjoining 1 is as follows.

Definition 2.2. Let $R$ be left faithful. Put $\kappa\left(\operatorname{End}\left(R_{R}\right)\right)=K$. Then $R^{1}$ is defined to be $R+K$, as a subring of $\operatorname{End}\left(R_{R}\right)$.

PROPOSITION 2.3. The characteristic ring $\kappa\left(R^{*}\right) \cong \kappa(R)$. If $R$ is left faithful, $\kappa\left(\operatorname{End}\left(R_{R}\right)\right)=\kappa\left(R^{1}\right) \cong \kappa(R)$, and $R^{1}$ is a homomorphic image of $R^{*}$.

Proof. These statements say that the two ways of adjoining 1 do not do much damage to the additive structure of $R$.

The first part for $R^{*}$ is clear. For $R^{1}$ we construct the function $g: \mathbf{P} \rightarrow$ $\mathbf{N} \cup\{\infty\}$ and see that it coincides with the function $f$ for $R$. Note also that $\operatorname{ann}_{\mathrm{Z}} R=\operatorname{ann}_{\mathrm{Z}} R^{1}$ since if $z R=0$ and $z \in \mathbf{Z}$, then $z 1=0$ in $R^{1}$ and conversely. Choose $p \in \mathbf{P}$. If $f(p)=i \neq \infty$ then $R=t_{p}(R) \oplus R^{(p)}, R^{(p)}$ 
$p$-divisible, and any $f \in \operatorname{End}\left(R_{R}\right)$ sends $t_{p}(R)$ to itself and $R^{(p)}$ to itself. Hence there is a central idempotent $e_{p} \in \operatorname{End}\left(R_{R}\right)$ with $e_{p}\left(R^{(p)}\right)=0$ and $e_{p}$ is the identity on $t_{p}(R)$. Now if for $y \in R^{1}, p^{k} y=0$ for some $k \geq 1$ then $y R \subseteq t_{p}(R)$ and so $p^{i} y=0$. Hence ann $t_{p}\left(R^{1}\right)=\left(p^{i}\right)$. Further, if $y \in t_{p}\left(R^{1}\right)$ then $e_{p} y=y$ giving $t_{p}\left(R^{1}\right)=e_{p} R^{1}$. Now if $0 \neq s \in\left(1-e_{p}\right) R^{1}$, define $\frac{1}{p} s$ by $\left(\frac{1}{p} s\right)(r)=\frac{1}{p} s(r)$, and this makes sense since $\left(1-e_{p}\right) R$ is $p$-divisible and $p$-torsion free. The conclusion is that $g(p)=f(p)$. If now $f(p)=\infty$ suppose that $R^{1}=t_{p}\left(R^{1}\right) \oplus U$ where $\operatorname{ann}_{\mathrm{Z}} t_{p}\left(R^{1}\right)=\left(p^{i}\right)$ and $U$ is $p$-divisible. Then there is an idempotent $e_{p}$ giving the splitting. Now $\frac{1}{p}\left(1-e_{p}\right)$ makes sense in $R^{1}$ and so $\left(1-e_{p}\right) R$ is $p$-divisible. Moreover, $t_{p}(R)=t_{p}\left(R^{1}\right) \cap R=e_{p} R$ and $R=e_{p} R \oplus\left(1-e_{p}\right) R$, which contradicts the assumption that $f(p)=\infty$.

The result for $\operatorname{End}\left(R_{R}\right)$ is implicit in the above.

Finally, define $\zeta: R^{*} \rightarrow R^{1}$ by $\zeta(r, z)=r+z$. This is clearly well-defined and surjective.

The kernel of the homomorphism $\zeta$ is $L:=\{(r, z) \mid$ for all $s \in R, r s=$ $-z s\}$, in other words, dividing by $L$ gets rid of any superfluity, that is, those elements of $\kappa(R)$ whose action is already present in $R$. If $R$ already had an identity element $e$ then $L$ would be generated by $(e,-1)$ and $R^{*} / L \cong R$.

We next look at some basic properties of $R^{*}$ and $R^{1}$. The centre of a ring $S$ will be denoted by $Z(S)$ and its right singular ideal by $\Sigma_{r}(S)$. The first observation is obvious.

LEMMA 2.4. The centre $Z\left(R^{*}\right)=\{(r, s) \mid r \in Z(R)\}$ and, if $R$ is left faithful, $Z\left(R^{1}\right)=\{r+s \mid r \in Z(R)\}$.

Proposition 2.5. If $R$ is left faithful, the ideal $R$ of $R^{1}$ is dense as a right ideal. Further

(i) $\Sigma_{r}(R)=0$ if and only if $\Sigma_{r}\left(R^{1}\right)=0$,

(ii) $R$ and $R^{1}$ have the same right uniform dimension,

(iii) $R$ is prime (semiprime) if and only if $R^{1}$ is prime (semiprime),

(iv) $Q_{r \max }(R)=Q_{r \max }\left(R^{\mathbf{1}}\right)$,

(v) $R$ is a semiprime Goldie ring if and only if $R^{1}$ is semiprime Goldie, and, in this case, $Q_{r c l}(R)=Q_{r c l}\left(R^{1}\right)$.

Proof. Robson [14, Proposition 1.2] has observed, in a more general setting, that $R$ is an essential right ideal of $R^{1}$. The stronger property of density is also easy to check. If $r+z, r^{\prime}+z^{\prime} \in R^{1}, r+z \neq 0$, then for some $s \in R$, $(r+z)(s) \neq 0$ and $r^{\prime} s+z^{\prime} s \in R$. The remainder of the proposition follows immediately. 
Note that $R$ need not be right essential in $R^{*}$. For example if $R=2 Z$ then $\kappa(R)=\mathbf{Z}$ and $(2,-2)$ has no non-zero multiple in $R$.

\section{Finiteness conditions}

In this section we examine finiteness conditions on $R, R^{*}$ and $R^{1}$.

Proposition 3.1. (i) If $R$ is (right or left) noetherian, so are $R^{*}$ and $R^{1}$.

(ii) If $R$ is (right or left) artinian and $R$ has no additive subgroup which is a quasicyclic group, then $R^{*}$ and $R^{1}$ are artinian.

Proof. (i) If $R$ is (right or left) noetherian, it suffices to show that $\kappa(R)$ is noetherian. Of the rings of (1.1), the first two types are noetherian. Suppose $\kappa(R)$ has the third form (that is, $\mathbf{X}_{1}$ is infinite). Then $t(R)$, which is an ideal, is an infinite direct sum of non-zero ideals, which is impossible.

(ii) If $R$ is (right or left) artinian, again we see that $\kappa(R)$ cannot be of the third type. If $\operatorname{ann}_{\mathrm{Z}} R=I \neq 0$ then $\kappa(R)=\mathrm{Z} / I$ is artinian. Moreover, if $\operatorname{ann}_{\mathrm{Z}} R=0, t(R)$ has only finitely many non-zero components and $\kappa(R)=$ $D \times \mathbf{Z} /\left(p_{1}^{f\left(p_{1}\right)}\right) \times \cdots \times \mathbf{Z} /\left(p_{k}^{f\left(p_{k}\right)}\right)$ as in (1.1). We need to show that $D=\mathbf{Q}$, and this follows immediately from [7, Theorem 73.1].

If $R$ is artinian and left faithful, then $R$ has no additive subgroups which are quasicyclic [7, Lemma 72.3] so the proposition applies in this case. In fact, Fuchs [7, Theorem 73.2] shows that an artinian ring $R$ can be embedded in an artinian ring with identity if and only if $R$ has no additive subgroups which are quasicyclic. Note that for $\mathbf{Z}_{p^{\infty}}$, the zero ring on a quasicyclic group, $\kappa\left(\mathbf{Z}_{p^{\infty}}\right)=\mathbf{Z}$.

At this point it is appropriate to mention Krull dimension. Of the three kinds of rings which appear as $\kappa(R)$, the first is artinian and hence of Krull dimension 0 , the second is not artinian but the torsion part is artinian and so is every proper factor of the torsion free part; it is of Krull dimension 1, the third type does not have finite uniform dimension and so does not have Krull dimension [11, Proposition 1.4].

Proposition 3.2. Let $R \neq 0$ be left faithful and have Krull dimension. Then so does $R^{1}$ and $K-\operatorname{dim} R=K-\operatorname{dim} R^{1}$.

Proof. If $K-\operatorname{dim} R=0$, this is the result for artinian rings. If $K$-dim $R=$ $\alpha>0$, then $R$ has finite uniform dimension and so $\kappa(R)$ is artinian with 
$K$-dim 0 or is of type (ii) with $K$-dim 1 , as remarked above. Hence $K$-dim $R^{1}=$ $\max \left(K-\operatorname{dim}\left(R^{1} / R\right), K-\operatorname{dim} R\right)=\alpha[11$, Lemma 1.1].

\section{Regularity and generalizations}

There are many results in the literature of the following form: let $R$ be a ring, not necessarily with 1 , which satisfies condition (\#), where (\#) is some generalization of (von Neumann) regularity; then $R$ can be embedded in a ring $R^{1}$ with 1 which also satisfies (\#). We will show, at least when $R$ is left faithful (a condition which sometimes comes free with (\#)), that these all can be achieved with our ring $R^{1}$. It will be shown at the end of this section that for all these sorts of regularity, $R^{1}$ satisfies a universal property with respect to adjoining 1 . We begin with a very general relative of regularity.

LEMMA 4.1. Let $R$ be such that every ideal is idempotent, that is, if I is an ideal then $I^{2}=I$. Then $\kappa(R)$ is regular.

Proof. (i) If $\operatorname{ann}_{\mathrm{Z}} R=(m) \neq 0$ then $m$ must be square-free and $\kappa(R) \cong$ $\mathbf{Z} /(m)$ is regular.

(ii) We need to show that the function $f$ which defines $\kappa(R)$ has $f(p) \leq 1$ for all $p \in \mathbf{P}$, for then $\kappa(R)$ will be regular. Pick a prime $p$. Consider $p R$. Since $p R=p^{2} R^{2}=p^{2} R, p R$ is $p$-divisible. Further, $p t_{p}(R)=p^{k} t_{p}(R)$ for all $k \geq 1$. This means that $p t_{p}(R)$ is both $p$-torsion and $p$-divisible. Let $x \in p t_{p}(R)$. For some integer $n \geq 1, p^{n} x=0$. Since $p t_{p}(R)=p^{n} t_{p}(R)$, $x p t_{p}(R)=0$. Hence $\left(p t_{p}(R)\right)^{2}=0$ and so, since $p t_{p}(R)$ is idempotent, $p t_{p}(R)=0$.

The following result unifies the known facts that regular rings [8] and rings with all ideals idempotent [6] can be appropriately embedded in rings with 1.

PROPOSITION 4.2. Let $R$ be regular, strongly regular or have all ideals idempotent. Then $R^{1}$ is likewise.

Proof. In each case $\kappa(R)$ is commutative regular and so satisfies all of the conditions. All that needs to be done is to verify that if $(C)$ is one of the conditions and $R$ is an ideal in a ring $S$ where $R$ and $S / R$ satisfy (C), then so does $S$. For regular rings this is [10, Lemma 1.3] and a similar proof works for strong regularity.

Finally it is easy to check that the class of rings all of whose ideals are idempotent is closed under extensions. 
There remain three important cases which need separate treatment: $\pi$ regular, biregular and right $V$-rings. The first is already in Fuchs and Rangawamy [9]; the ring constructed there is precisely $R^{*}$. In this case there is no analogue of the "universal" regular ring of Fuchs and Halperin [8] which, incidentally, maps onto $\kappa(R)$ for all regular rings $R$.

Proposition 4.3 [9]. Let $R$ be $\pi$-regular. Then $R$ can be embedded in a $\pi$-regular ring if and only if $R^{*}$ is $\pi$-regular which is if and only if, for each prime $p, \operatorname{ann}_{\mathrm{Z}} t_{p}(R) \neq 0$ and $R / t(R)$ is $p$-divisible. In this case $R^{1}$ is also $\pi$-regular.

Proof. The necessity is [9, Lemma 2]. The ring constructed in [9, Theorem 2] is what we have called $R^{*}$ and $\pi$-regularity is preserved under homomorphic images.

The fact that a biregular ring can be embedded in a biregular ring with 1 was established by Vrabec in [18]. Here the proof is simplified and we observe that the adjunction of 1 can be achieved via $\kappa(R)$.

Proposition 4.4. Suppose we have a ring $S$ with identity element containing an ideal $R$ which, as a ring, is biregular. Suppose $S$ also has a central regular subring, $Z$, such that $S=Z+R$. Then $S$ is biregular.

Proof. Since biregular rings with 1 are precisely the rings whose Pierce sheaves have simple stalks [4, Propositions 2.13 and 2.19], it suffices to show that for every maximal ideal $M$ of $B$, the boolean algebra of central idempotents of $S, M S$ is a maximal ideal of $S$. (However, that this is sufficient can be seen directly, without reference to the sheaf structure: $S$ is clearly semiprime and consider, for $a \in S, S a S \oplus \operatorname{lann} S a S$; if this is not $S$ it is contained in a maximal ideal $N=(N \cap B) S$. Write $a=a e, e \in N \cap B$; then $1-e \in \operatorname{lann} S a S \subseteq N$, which is impossible.) Suppose, then, that $M$ is maximal in $B$. Suppose $K$ is an ideal of $S$ with $M S \subset K$. If $M S \cap R \neq K \cap R$, then, since $R$ is biregular, there is $e \in(B \cap K) \backslash M S$. Thus $1-e \in M \subseteq K$ and so $K=S$. Now consider the case where $M S \cap K=K \cap R$. Since $M S \subset K, M S+R \subset K+R$. If $M S \cap R \neq R$, since $R$ is biregular there is $e \in(B \cap R) \backslash M S$. Hence $1-e \in M$, forcing $M S+R=S$. This contradicts the fact that $M S+R \subset K+R$, so $R \subseteq M S$. It follows that there is a $z \in(Z \cap K) \backslash M S$ and so, since $Z$ is regular, an $e \in(B \cap K) \backslash M S$. Then $1-e \in M S \subseteq K$ and, finally, $K=S$.

COROLlary 4.5. If $R$ is biregular so are $R^{*}$ and $R^{1}$. 
Proof. We need only remark that $\kappa(R)$ is regular by (4.1). Then (4.4) gives the result.

Recall that a ring $S$ is a right $V$-ring if every simple right $S$-module is injective. That is, if $M$ is module such that $M S \neq 0$ and 0 and $M$ are its only submodules, then $M$ is injective. If $S$ is a ring with 1 then it is a right $V$-ring if and only if every unital $S$-module $M$ satisfies $J(M)=0$ where $J(M)=\bigcap\{N \mid N$ is a maximal submodule of $M\}$ [13, Theorem 2.1]. If, now, $R$ is any ring such that $A R=A$ for any right ideal $A$, then $R$ is an right $V$-ring if and only if any right ideal $A$ of $R$, with $A \neq R$, is an intersection of maximal modular right ideals (Villmayor $[12,2.24]$ ). Our first observation is about $\kappa(R)$. Recall that a commutative ring is regular if and only if it is a $V$-ring (this is due to Kaplansky [15, Theorem 6]).

LEMMA 4.6. If $R$ is a right $V$-ring such that $A R=A$ for all right ideals $A$ of $R$, then $\kappa(R)$ is regular.

Proof. As in (4.1) we show that for all $p \in \mathbf{P}, f(p) \leq 1$.

Take $p \in P$. Then $p R=R$ would show that $f(p)=0$. Otherwise it will turn out that $p R$ is $p$-divisible. If not, there is a maximal modular ideal $M$ such that $p^{2} R \subseteq M$ and $p R \not \subset M$. Then $p R+M=R$, and multiplication by $p$ gives the contradiction $p^{2} R+p M=p R$ and $p R \subseteq M$. Staying in the situation where $p R \neq R$, consider $t_{p}(R)$. Suppose $x \in t_{p}(R)$ and $p x \neq 0$; then there is $k \geq 2$ such that $p^{k-1} x \neq 0$ but $p^{k} x=0$. Let $T$ be the right ideal generated by $p^{k-1} x$. There is a maximal modular ideal $M \neq R$ such that $p^{k-1} x \notin M$. Then $T+M=R$. Thus $p M=p R$ and so $p^{k-1} x=p\left(p^{k-2} x\right) \in p M \subseteq M$. This contradiction shows that $p t_{p}(R)=0$. Finally, for $r \in R, p r=p^{2} s$ for some $s$, and so $p(r-p s)=0$. Hence $r=p s+(r-p s) \in p R \oplus t_{p}(R)$. In summary, in this case $f(p)=1$.

LEMma 4.7. Suppose that $R$ is an ideal in a right $V$-ring $S$ with identity element. Then for any right ideal $A$ of $R, A R=A$.

Proof. Let $A$ be a right ideal of $R$ and suppose that $A R \neq A$. Since the $S$-module $S / A R$ satisfies $J(S / A R)=0$, there is a maximal $S$ submodule $T$ of $S$ with $A R \subseteq T$ but $A \not \subset T$. Then $A S+T=S$ and so multiplying by $R$ we obtain $A R+T R=R$. Thus $R \subseteq T$ and so $A \subseteq T$. This contradiction shows that $A R=A$.

Proposition 4.8. Suppose that $S$ is a ring with 1 and $R$ an ideal of $S$ such that $A R=A$ for all right ideals $A$ of $R$; suppose further that $R$ and $S / R$ are right $V$-rings. Then $S$ is a right $V$-ring. 
Proof. Let $I$ be a right ideal of $S$ and suppose that $a \in S \backslash I$. We must show that there is a maximal right ideal $M$ of $S$ such that $I \subseteq M$ and $a \notin M$. We first consider the case where $a \in I+R$. Then $a=b+r$ where $b \in I$ and $r \in R \backslash I$. If $J$ is a right ideal of $S$ with $I \subseteq J$, then $a \notin J$ if and only if $r \notin J$. Thus we may assume that $a \in R$. Let $N$ be a maximal modular right ideal of $R$ such that $I \cap R \subseteq N$ and $a \notin N$. Let $e \in R$ be such that $e r-r \in N$ for all $r \in R$. Define $\phi: S \rightarrow R / N$ by $\phi(s)=r+e u$ if $s=r+u, r \in R$. It is straightforward to check that $\phi$ is a well-defined right $S$-map; $R / N$ is naturally a simple right $S$-module because $N R=N$. The kernel of $\phi$ is the desired maximal right ideal of $S$. Further, if $s=r+u, r \in R$, is in $I$, then $e(r+u) \in N$. Hence $I \subseteq \operatorname{ker} \phi$ since $e r-r \in N$ for all $r \in R$.

There remains the case where $a \notin I+R$. But since $S / R$ is a $V$-ring and $a+R \notin(I+R) / R$ there is a maximal right ideal $M$ of $S$ such that $I+R \subseteq M$ and $a \notin M$.

Proposition 4.9. For a ring $R, R^{*}$ is a right $V$-ring if and only if $R$ is a right $V$-ring and for every right ideal $A$ of $R, A R=A$. In this case, $R^{1}$ is also a right $V$-ring.

Proof. Suppose first that $R^{*}$ is a right $V$-ring. By (4.7), $A R=A$ for all right ideals $A$ of $R$. Let $A$ be a right ideal of $R$. It is also a right ideal of $R^{*}$ so there are maximal right ideals $\left\{M_{\alpha}\right\}$ such that $A=\cap M_{\alpha}$. It suffices to show that for any $\alpha$ with $M_{\alpha} \cap R \neq R, N_{\alpha}=M_{\alpha} \cap R$ is maximal modular in $R$. It is clearly maximal. Further, $M_{\alpha}+R=R^{*}$ so there are $e \in R, a \in M_{\alpha}$ with $1=a+e$ and so $e-e^{2}=a e \in N_{\alpha}$ and for all $r \in R, r-e r=a r \in N_{\alpha}$.

The converse is immediate from (4.6) and (4.8).

It can now be shown that in the context of (generalized) regularity, our way of adjoining 1 satisfies a universal property.

THEOREM 4.10. Let $g: R \rightarrow S$ be a monomorphism where

(i) $S$ is a ring with 1 ,

(ii) the identity of $S$ is contained in a $\pi$-regular subring of $S$,

(iii) $g(R)$ is an ideal of $S$,

(iv) if $s \in S$ is such that $s g(R)=0$, then $s=0$.

Then there is a unique homomorphism $\bar{g}: R^{1} \rightarrow S$ such that $\bar{g} \pi=g$ where $\pi: R \rightarrow R^{1}$ is the usual embedding: $\pi(r) \in$ End $R_{R}$ is defined by $\pi(r)(t)=r t$.

Proof. We first check that $R$ and $S$ have the same characteristic ring. If $n R=0$ for some $n>0$, then $(n S) g(R)=0$ and so $n S=0$. Thus $\kappa(R)=\kappa(S)$ in this case. 
Now suppose that $\kappa(R)$ is given by $f_{R}$ and that $f_{R}(p)=k \neq \infty$. Then $\operatorname{ann}_{\mathbf{Z}}\left(t_{p}(R)\right)=\left(p^{k}\right)$ and $R=t_{p}(R) \oplus R^{(p)}$ where $R^{(p)}$ is $p$-divisible. Let $s \in t_{p}(S)$. Then $p^{k} s g(R)=0$ and so $p^{k} s=0$. Hence $\operatorname{ann}_{\mathbf{Z}}\left(t_{p}(S)\right)=\left(p^{k}\right)$. Also, $\left(t_{p}(S) \cap p^{k} S\right) g(R) \subseteq t_{p}(g(R)) \cap p^{k} g(R)=0$ because $R^{(p)}=p^{k} R$, and so $t_{p}(S) \cap p^{k} S=0$. Since $p^{k}$ is contained in some $\pi$-regular subring of $S$, there is $n \geq 1$ and $s \in S$ such that $p^{2 n k} s=p^{n k}$. Thus, $p^{k}-p^{k+1} s^{\prime} \in t_{p}(S) \cap p^{k} S=0$ for some $s^{\prime} \in S$, and so $p^{k}=p^{k+1} s^{\prime}$. It follows that $p^{k} S$ is $p$-divisible. Now if $s \in S, p^{k} s=p^{k+1} t$ for some $t \in p^{k} S$ and so $s=(s-p t)+p t \in t_{p}(S)+p^{k} S$. Hence $S=t_{p}(S) \oplus p^{k} S$ where $p^{k} S$ is $p$-divisible. Note that the case $k=0$ is included with the appropriate adjustment of notation.

Conversely, suppose that $\kappa(S)$ is given by $f_{S}$ and that $f_{S}(p)=n \neq \infty$. Thus $S=t_{p}(S) \oplus S^{(p)}$ where $S^{(p)}$ is $p$-divisible. Then $p s=1-e_{p}$ for some $s \in S^{(p)}$, where $e_{p}$ is the central idempotent giving $t_{p}(S)$. Put $R^{(p)}=\left(1-e_{p}\right) g(R)$. Then $s R^{(p)} \subseteq R^{(p)}$ since $R^{(p)}$ is an ideal of $S$. But $p s R^{(p)}=\left(1-e_{p}\right) R^{(p)}=R^{(p)}$ and so $s R^{(p)}=R^{(p)}$, which shows that $R^{(p)}$ is $p$-divisible, and clearly $g(R)=$ $R^{(p)} \oplus t_{p}(g(R))$.

We have shown that $\kappa(R) \cong \kappa(S)$. Let $\phi: S \rightarrow$ End $R_{R}$ be defined by $\phi(s)(r)=g^{-1}(s g(r))$. Clearly $\phi(s) \in$ End $R_{R}$. Since we know (by (1.2)) that $\phi(\kappa(S)) \subseteq \kappa\left(\operatorname{End}\left(R_{R}\right)\right) \cong \kappa(R)$, the above shows that $\phi(\kappa(S))=\kappa\left(\operatorname{End}\left(R_{R}\right)\right)$. Define $\bar{g}: R^{1} \rightarrow S$ by $\bar{g}(\phi(t)+\pi(r))=t+g(r)$ where $t \in \kappa(S), r \in R$. We check that $\bar{g}$ is well defined. If $\phi(t)+\pi(r)=\phi\left(t_{1}\right)+\pi\left(r_{1}\right)$, then $(\phi(t)+\pi(r))(\alpha)=$ $\left(\phi\left(t_{1}\right)+\pi\left(r_{1}\right)\right)(\alpha)$ for all $\alpha \in R$. Thus $g^{-1}(t g(\alpha))+r \alpha=g^{-1}\left(t_{1} g(\alpha)\right)+r_{1} \alpha$ and so $t g(\alpha)+g(r \alpha)=t_{1} g(\alpha)+g\left(r_{1} \alpha\right)$ for all $\alpha \in R$. Hence $(t+g(r)) g(\alpha)=\left(t_{1}+\right.$ $\left.g\left(r_{1}\right)\right) g(\alpha)$ for all $\alpha \in R$ and so $t+g(r)=t_{1}+g\left(r_{1}\right)$. Now it is straightforward to check that $\bar{g}$ is a ring homomorphism and it obviously satisfies $\bar{g} \pi=g$.

Finally we check that $\bar{g}$ is unique. Suppose that $h, k: R^{1} \rightarrow S$ are ring homomorphisms such that $k \pi=h \pi=g$. If $x \in R^{1}, r \in R$ then since $\pi(R)$ is an ideal of $R^{1}$ there exists $a \in R$ such that $x \pi(r)=\pi(a)$. Now $h(x) g(r)=$ $h(x) h \pi(r)=h(x \pi(r))=h(\pi(a))=g(a)$ and $k(x) g(r)=k(x) k \pi(r)=$ $k(x \pi(r))=k(\pi(a))=g(a)$. Then $(h(x)-k(x)) g(R)=0$ and, finally, $h(x)=k(x)$ for all $x \in R^{1}$.

COROLlaRy 4.11. Let $S$ be a ring with identity and $g: R \rightarrow S$ a monomorphism, where $R$ is left faithful and $g(R)$ is an ideal of $S$, essential as an ideal. If $R$ and $S$ are both regular (biregular, have all ideals idempotent, $\pi$ regular, respectively) then there is a unique homomorphism $\bar{g}: R^{1} \rightarrow S$ such that $\bar{g} \pi=g$.

Note that the condition that $s g(R)=0$ implies $s=0$ (or, in the corollary, that $R$ be left faithful) cannot be omitted: consider $2 Z /(4) \rightarrow Z /(4)$. 


\section{Algebras over Dedekind domains}

The point of view throughout has been to view rings as Z-algebras. However (1.1) is true for epimorphs of any Dedekind domain $D$ if, instead of prime elements, we take maximal ideals. Since every proper homomorphic image of $D$ is artinian, all the arguments above will hold just as well in this more general setting.

\section{References}

[1] A. K. Bousfield and D. M. Kan, 'The core of a ring', J. Pure Appl. Algebra 2 (1972), 73-81.

[2] W. D. Burgess and P. Menal, 'Strongly $\pi$-regular rings and homomorphisms into them', Comm. Algebra 16 (1988), 1701-1725.

[3] T. Cheatham and E. Enochs, 'The epimorphic images of a Dedekind domain', Proc. Amer. Math. Soc. 35 (1972), 37-42.

[4] J. Dauns and K. H. Hofmann, 'The representation of biregular rings by sheaves', Math. $Z$. 91 (1966), 103-123.

[5] W. Dicks and W. Stephenson, 'Epimorphs and dominions of Dedekind domains', J. London Math. Soc. 29 (1984), 224-228.

[6] S. Feigelstock, 'An embedding theorem for weakly regular and fully idempotent rings', Comment. Math. Univ. St. Paul 27 (1978), 101-103.

[7] L. Fuchs, Abelian groups (Pergamon Press, Oxford, 1960).

[8] L. Fuchs and $I$. Halperin, 'On the imbedding of a regular ring in a regular ring with identity', Fund. Math. 54 (1964), 285-290.

[9] L. Fuchs and K. M. Rangaswamy, 'On generalized regular rings', Math. Z. 107 (1968), 71-81.

[10] K. R. Goodearl, Von Neumann regular rings (Pitman, London, 1979).

[11] R. Gordon and J. C. Robson, 'Krull dimension', Mem. Amer. Math. Soc. 133 (1973).

[12] K. Koh, Quasisimple modules and other topics in ring theory (Lecture Notes in Math. 246 (1972), 323-428).

[13] G. Michler and O. Villamayor, 'On rings whose simple modules are injective,' J. Algebra 25 (1973), 185-201.

[14] J. C. Robson, 'A unified approach to unity,' Comm. Algebra 7 (1979), 1245-1255.

[15] A. Rosenberg and D. Zelinsky, 'On the finiteness of the injective hull,' Math. Z. 70 (1959), 372-380.

[16] H. H. Storrer, 'Epimorphismen von kommutativen Ringen', Comment Math. Helv. 43 (1968), 378-401.

[17] H. H. Storrer, 'Epimorphic extensions of non-commutative rings', Comment. Math. Helv. 48 (1973), 72-86.

[18] J. Vrabec, 'Adjoining a unit to a biregular ring', Math. Ann. 188 (1970), 219-226.

Department of Mathematics

University of Ottawa

Ottawa, Ontario

Canada K1N 6N5
Department of Mathematics, Statistics and Computing Science Dalhousie University Halifax, Nova Scotia Canada B3H 3J5 